\title{
The evolution of black hole states
}

\author{
Jeroen Homan \\ MIT Center for Space Research \\ 70 Vassar Street, Cambridge, MA 02139, USA \\ jeroen@space.mit.edu \\ Tomaso Belloni \\ INAF/Osservatorio astronomico di Brera \\ Via E. Bianchi 46, 23807, Merate (LC), Italy \\ belloni@merate.mi.astro.it
}

\begin{abstract}
We discuss the evolution of black hole transients on the basis of a few systems that were intensively observed with the Rossi X-ray Timing Explorer. We focus on the global evolution and the observed state transitions. Rather than giving a numerical recipe for classifying observations, we try to identify times during outbursts at which clear changes occur in the X-ray variability, X-ray spectral, or multi-wavelength properties.
\end{abstract}

\section{Introduction}

Since the launch of the Rossi X-ray Timing Explorer (RXTE) about $\sim 20$ black hole X-ray transients have been observed with enough coverage to study their global evolution. These observations have provided a wealth of information and have already led to a considerable increase in our understanding of these systems. In a recent review by McClintock and Remillard (2004) a new classification scheme was proposed for the spectral and variability states as observed in black hole transients. McClintock and Remillard (2004) approached the states issue by identifying three 'stable' states in black hole transients. In this paper we approach the issue from an other angle, focusing on the observed transitions, the overall evolution during an outburst, and how X-ray changes relate to changes at other wavelengths.

\section{State definitions}

Until the late 1990s it was generally assumed that the state of a blackhole system was determined by the instantaneous mass accretion rate, $\dot{M}$. It was believed that as the mass accretion rate increased, a source went through the following states (see e.g. Esin et al., 1997) : quiescence $\rightarrow$ low/hard $\rightarrow$ intermediate $\rightarrow$ high/soft $\rightarrow$ very high. Observations with RXTE of sources like XTE J1550-564 have shown that such

(C) 2018 Kluwer Academic Publishers. Printed in the Netherlands. 
a simple $\dot{M}$-driven picture, with transitions only being triggered by changes in $\dot{M}$, is probably not able to explain the observed transition between the states. Homan et al. (2001) suggested that an additional parameter may play a role in those transitions. They also suggested that the very-high state and the intermediate state(s) are one and the same state and represent transitions between the low/hard and high/soft states at different luminosities. The fact that most of the states were observed over wide and overlapping ranges in luminosity also meant that attributes like 'low', 'high' and 'very high' had lost most of their significance.

McClintock and Remillard (2004) introduced a new classification scheme that is partly based on the old five-states scheme, but no longer uses luminosity as a selection criterion since it appeared that any of the active states may occur at any luminosity. They still recognize a quiescent state, hard state, soft state (renaming the latter the 'thermal dominant state'), and very-high state (renaming it the steep power-law state), but drop the intermediate state as a bona fide state. The three active states are defined on the basis of the fractional contribution of the disk-flux (or power-law flux) to the $2-20 \mathrm{keV}$ spectrum, spectral power-law index, and the strength of the power continuum. All observations that cannot be classified according to their state definitions, are combined into an intermediate state, in which observations can show properties of any of the three main states.

Some of the state transitions discussed in this paper involve (and are defined on the basis of) sudden changes in the properties of the quasi-periodic oscillations (QPOs) in the $\sim 1-10 \mathrm{~Hz}$ range. Currently three types of these low-frequency QPOs are recognized in black hole binaries, called type A, B and C (Wijnands et al., 1999, Remillard et al., 2002). The three types can be distinguished on the basis of strength, coherence, phase lags, energy dependence, harmonic content, and frequency stability on a time scale of days. Type C QPOs are the most common ones; they are observed in the spectrally hard states over a wide range in frequency $(\sim 0.1-10 \mathrm{~Hz})$, and are stronger and more coherent than the other two types. Type A and B QPOs have only been observed in a few sources; they are only seen in the veryhigh/steep power-law state, in a narrow frequency range $(\sim 4-8 \mathrm{~Hz})$. Recent observations of H1743-322 (Homan et al., 2004b) suggest that these two types might be more intimately related than was previously believed. Transitions from one type of QPO to another always seem to involve type B (Casella et al., 2004), with transitions between type C and $\mathrm{B}$ resulting in a clear change in the shape and strength of noise continuum. The main reason for including QPO type in our discussion of black hole states is that they provide an additional indication for 
changes in the accretion flow that may not always show up as strong spectral transitions.

\section{Global evolution}

Hardness-intensity diagrams (HIDs), in which the X-ray count rate is plotted versus an X-ray color, provide a quick way to study the global evolution of black hole transients during outburst. In Figure 1 we plot the light curves and HIDs of four transients that were observed with $R X T E$ between 1999 and 2003. It should be noted that constant count rate levels in these HIDs correspond to substantially higher luminosities at hardness values close to 1 than at values of 0.001-0.01. The HIDs are similar to each other in that all four sources seem to trace out (part of) a counter-clockwise q-shaped track. Below a certain count rate the spectrum is always very hard (i.e. in the hard state) and above the spectrum is either very hard or very soft, except for two transitional phases (the 'horizontal' branches in the HID). This means, as was noted by other authors as well (see e.g. Maccarone and Coppi, 2003), that within a single outburst the hard $\rightarrow$ soft transition occurs at a flux that is about a factor of 10-100 higher than the soft $\rightarrow$ hard transition. Note that in GX 339-4 and XTE J1650-500 the hard $\rightarrow$ soft transition was rather fast, while in XTE J1859+226 and H1743-322 the source lingered for a longer time at intermediate colors.

While the sources shown in Figure 1 show a smooth overall movement through their HID, the fast time variability and multi-wavelength properties allow one to define a few rather sharp boundaries corresponding to state transitions. In the following, we will base our discussion of states mostly on the 2002/2003 outburst of GX 339-4, the results of which will presented in Belloni et al. (2004) and Homan et al. (in prep.). Complete references for our discussion can be found in those works.

\section{A detailed look at the states}

\subsection{The hard State - RISE AND DECAY}

Since pointed observations of a transient in outburst often start only when the source already has a luminosity that is a factor of more than $10^{4}-10^{5}$ above the quiescent level, not much is known about the early evolution of outbursts. Thanks to a monitoring campaign set up by David Smith and co-workers, the 2002/2003 outburst of of GX 339-4 

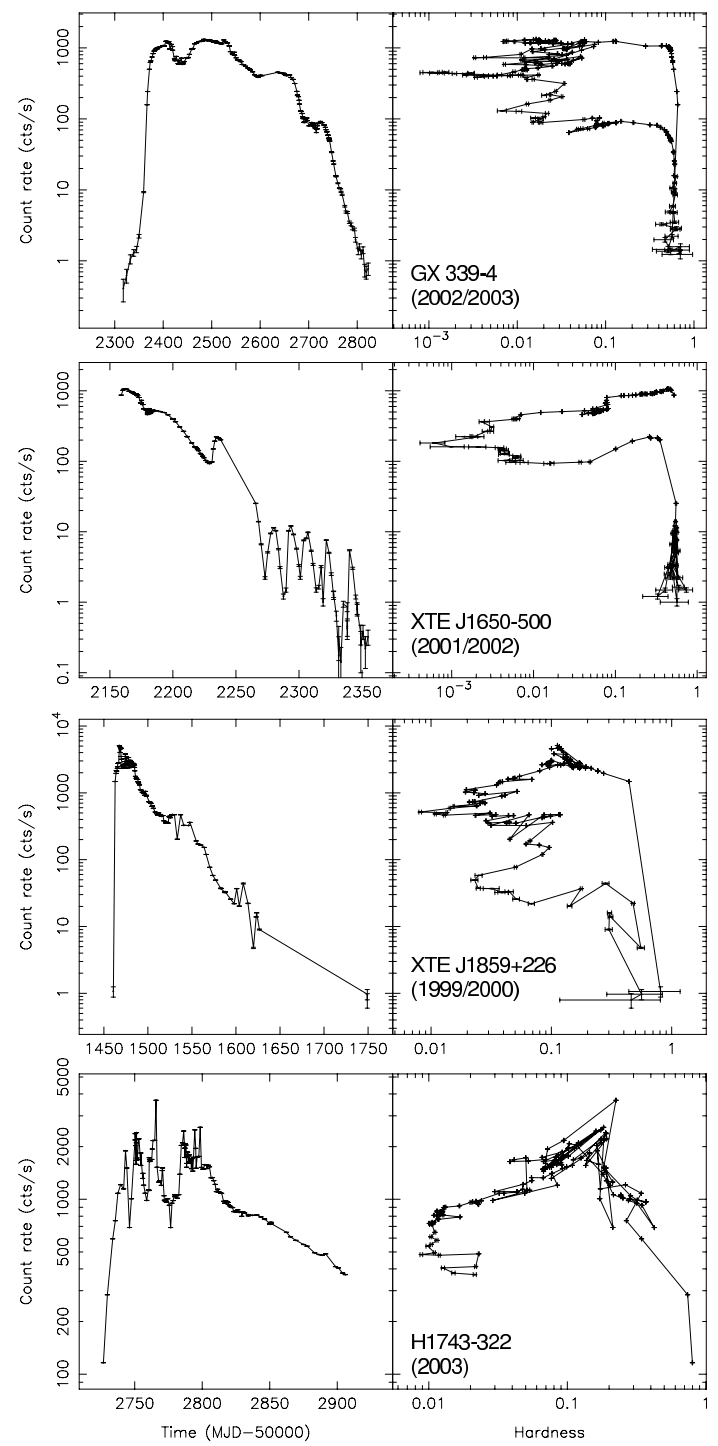

Figure 1. Light curves and hardness-intensity diagrams of four recent transients observed with the $R X T E / \mathrm{PCA}$. Count rates are in the $\sim 3-21 \mathrm{keV}$ band and hardness is defined as the ratio of count rates in the $\sim 6-19 \mathrm{keV}$ and $\sim 3-6 \mathrm{keV}$ bands. For GX 339-4, XTE J1859+226, and H1743-322, the outbursts were observed to start in the upper-right corner, with the sources moving through the diagram in a counter-clockwise direction. For XTE J1650-500 the initial rise was not observed with the PCA, but ASM observations suggest it started in the lower-right corner as well, following a vertical path to the upper-right corner, where the PCA coverage started.

was the first whose evolution could be followed from a flux level that was only a factor of 10 above its 2000 quiescent level (Corbel et al., 


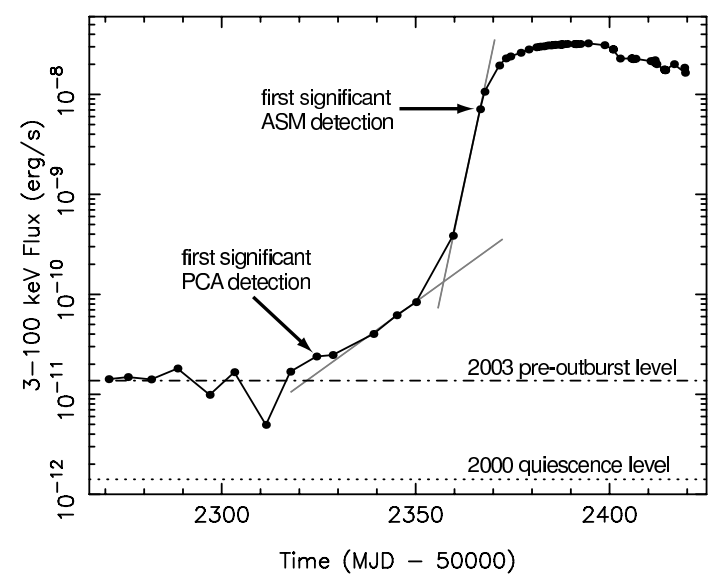

Figure 2. An RXTE light curve of the rise of the 2002/2003 outburst of GX 339-4. The times of the first significant PCA and ASM detections are indicated, as are the pre-outburst and quiescence levels. The two gray lines show the presence of two different time scales during the early rise.

2003). In Figure 2 we show the early evolution of GX 339-4. From this figure it is clear that before the first ASM detection the source luminosity had been increasing for at least 40 days, initially slowly and later more rapidly.

Throughout these early stages of the outburst, until it had reached its peak $3-100 \mathrm{keV}$ flux, the source was in the hard state: the strength of the broad-band variability decreased from $45 \% \mathrm{rms}$ to $30 \% \mathrm{rms}$ and the energy spectrum was dominated by a power law component, with the index slowly increasing from 1.3 to 1.4. During the rise the frequencies of the noise components and the occasional QPO increased gradually. An example of a power-density spectrum from this state in GX 339-4 is shown in Figure 3.

Just after reaching its peak flux, increases in the power-law index and noise/QPO frequencies accelerated. Also, a strong increase of the spectrally soft disk component and a substantial decrease in the optical/IR flux (see Fig. 4) were observed at that time, with the latter probably being the result of the jet switching off (or starting to, Homan et al., 2004a). At this point the source entered an intermediate state (see $\S 4.2$ ), with the transition from the hard state being visible as an almost $90^{\circ}$ turn to the left in the HID. Note that in the definition of McClintock and Remillard (2004) sources stay in the hard state until the power-law steepens beyond an index of 2.1. In our view the hard state is limited to only the hardest power-law dominated spectra with indices around 1.3-1.5, as many changes occur (simultaneously) when the power-law becomes steeper. 

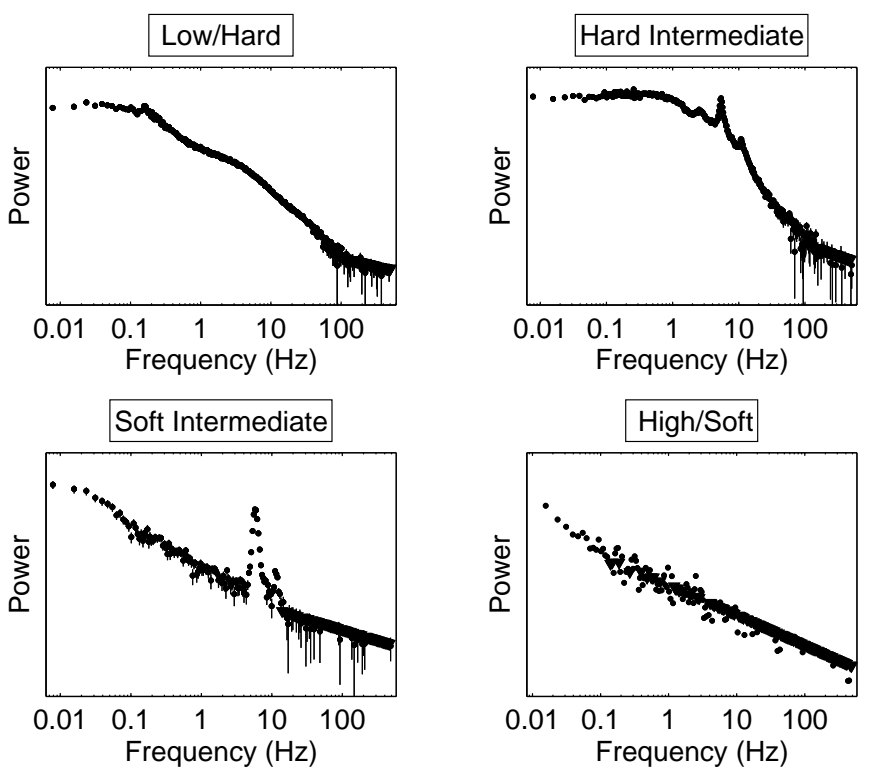

Figure 3. Examples of power-density spectra of GX 339-4 from the four states discussed in the text.

At the end of the 2002/2003 outburst GX 339-4 returned to the hard state, similar to what is observed in most, if not all, black hole transients. In the HID this can be nicely seen as the lower horizontal branch bending down to start running parallel to, and in fact nearly on top of, the hard state branch that was traced out during the rise. Such 'saturation' of the spectral hardness was also observed in XTE J1650-500 (Rossi et al., 2003, see also Fig. 1). It is interesting to note that once GX 339-4 reached the hardness at which it originally left the hard state branch, the optical/IR showed a strong increase (see light curves in Bailyn and Ferrara, 2004), indicating that the jet does not become visible in the optical/IR (or switch on) until the hard state branch is reached (as was already suggested by Kalemci et al., 2004 based on observations $4 \mathrm{U}$ 1543-47). The spectral hardening towards the hard state is not necessarily a monotonic process, as can be seen in the HID of GX 339-4 and XTE J1859+226.

Observations of hard state branches extending over many orders of magnitude in GX 339-4 and XTE 1650-500 indicate that the hard state accretion flow geometry (which likely gives rise to strong outflows) can exist over a wide range in mass accretion rate. 


\subsection{The Hard STATE $\leftrightarrow$ SOFT STATE TRAnsitions}

Immediately after leaving and before returning to the hard state, black hole transients are observed in an intermediate state, with spectral power-law indices between roughly 1.5-2.5. We are not aware of any exception to this. This state is different from the hard state in several aspects. Depending on whether the intermediate state is observed during the hard $\rightarrow$ soft or soft $\rightarrow$ hard transition, sources show a clear steepening/flattening of the spectral power law component, a strong increase/decrease in the noise and QPO frequencies, and an increasing/decreasing fractional disk flux, compared to the hard state. All these properties evolve smoothly from and to the hard state. In fact, without the HID and optical/IR information (see Fig. 4) it would be difficult to exactly pin-point the transition between these two states. In the case of the hard $\rightarrow$ soft transition the movement to the left in the HID is due to the combination of increased disk flux and steepening of the power law. In the timing domain, the broad-band variability components seen in the power density spectrum increase their characteristic frequencies, showing an evolution that clearly links them to the corresponding components in the hard state. A clear type C QPO appears, also with a characteristic frequency increasing with time and decreasing hardness (see Belloni et al. (2004)). A typical power-density spectrum from the intermediate state is shown in Figure 3.

It is important to note that the hard $\rightarrow$ intermediate state transition does not always occur at the same flux level, as can be seen from Figure 4. During the 2004 outburst of GX 339-4 this transition occurred at a flux level that was about a factor of 4 lower than in 2002. The transition in 2004 was preceded by a small hard state outburst, which suggests that the flux level at which the transition occurs depends on the recent accretion history. After passing through the intermediate GX 339-4 continued to brighten in the soft sate during its 2004 outburst.

Another important point to note is the fact that the occurrence of the intermediate state does not seem to depend on the time derivative of $\dot{M}$. In XTE J1650-500 it occurred during the decay, but in GX 3394 while $\dot{M}$ was apparently still increasing, as witnessed by the second (soft) maximum in the light curve. However, in both cases the transition resulted in a (temporary) drop in the count rate by a factor of $\sim 2$.

Taking once more the 2002/2003 outburst of GX 339-4 as a template, we see that when the hardness goes below a well defined threshold, the timing properties change sharply: a clear type B QPO appears in the power density spectrum (Figure 3, see also Casella et al., 2004 for similar behavior in XTE J1859+226). In most sources the change from type $\mathrm{C}$ to type B QPOs seems to take place when the power-law 

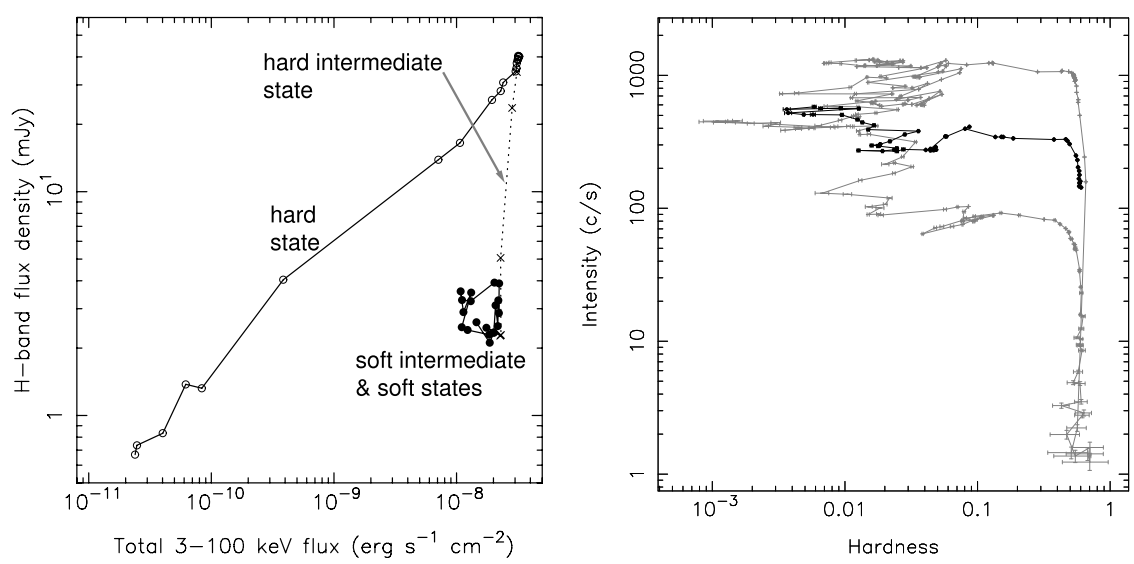

Figure 4. Left panel: X-ray/IR correlation for the first part of the 2002/2003 outburst of GX 339-4 (Homan et al., 2004). The different behavior for the different states is evident. Right panel: HID of the two most recent outbursts of GX 339-4: 2002/2003 (gray) and 2004 (black).

index has a value around 2.5-3.0. In fact, power-laws with such indices are accompanied by a great variety of timing properties: not only type A, B or C QPOs, but also very weak variability like that seen in the soft state.

We will refer to the part of the transition during which the power-law index changes between $\sim 1.5-2.5$ and which shows type $\mathrm{C}$ QPOs and strong band-limited noise as the hard intermediate state. The part of the transition where the power-law index is relatively constant around a value of 2.5-3.0 and during which the source occasionally shows type A and B QPOs on top of weaker red noise will be referred to as the soft intermediate state. Note that in GX 339-4 the soft intermediate state also showed observations with weak band limited noise and/or QPOs that we were not able to classify. It is therefore more a collection of different types of behavior, between which the source could switch on a time scale of a day, rather than a well defined state.

It is important to note that the change from the hard intermediate to the soft intermediate state was repeated again in the 2004 outburst at the same spectral hardness. During the 2002/2003 outburst a huge radio flare/ejection event was observed around the time of the change from the hard intermediate to the soft intermediate state (Gallo et al., 2004, Fender et al., 2004). Although it is tempting to associate such a flare with this transition, it should be mentioned that similar radio events are also observed in GRS 1915+105 (Belloni et al., 2000), a source for which characteristic type A and B QPOs were not observed to date. However, given the fast time scales of transitions in this system, 
it is possible that such QPOs appear for intervals shorter than for other sources, which would make them difficult to observe.

It is during the soft intermediate state that most high-frequency QPOs have been detected, indicating that there might be a relation between those and type A/B QPOs; indeed, the frequencies of both features are not observed to vary by a large amount between different observations. It is possible however that the high frequency QPOs in soft intermediate state evolve from broader features in the hard intermediate state, as is suggested by observations of XTE J1650-500 (Homan et al., 2003). Notice that while the soft intermediate state is often observed at high flux between the hard intermediate and the soft states, the same timing properties are not observed at low flux, when the reverse transition takes place. Moreover, in XTE J1550-564 (Homan et al., 2001) type A and B QPOs appeared at several well separated luminosity levels, strongly suggesting they are not strictly related to hard $\leftrightarrow$ soft transitions. In GX 339-4 a strong $1 \mathrm{~Hz}$ QPO with some properties similar to those of the type B QPOs is observed at low flux, indicating that it is possible that a similar state exist also at much lower accretion rate, although with different characteristic frequencies (Belloni et al., 2004).

\subsection{The Soft State}

During the soft state, which in GX 339-4 took place after the soft intermediate state, the spectral and timing properties are rather well defined, although a single clear transition from the soft intermediate state is hard to identify. The energy spectrum of the soft state is dominated by a strong thermal component, with the presence of a weak steep power-law component, which was not observed to show a highenergy cutoff (see Grove et al., 1998). Variability in the soft state is weak compared to the other states, with typical rms amplitudes of at most a few \% rms. Unfortunately, by the time most transients reach the soft state they are usually in the decay phase and observations have become shorter and less frequent, so detailed studies of its variability properties are rare. Nevertheless, a few weak QPOs have been detected in the soft states of GRO J1655-40, XTE J1550-564, and H1743-322. These QPOs all had frequencies that were higher than the other low frequency QPOs detected in those sources and the noise continua could all be fitted with broken power law. Surprisingly, the QPO and break frequencies of these power spectra fall on top of the Wijnands-van der Klis relation (Wijnands \& van der Klis 1999) for black holes in the hard and hard intermediate states. This suggests not only that these might be type C QPOs, but more importantly, that variability 


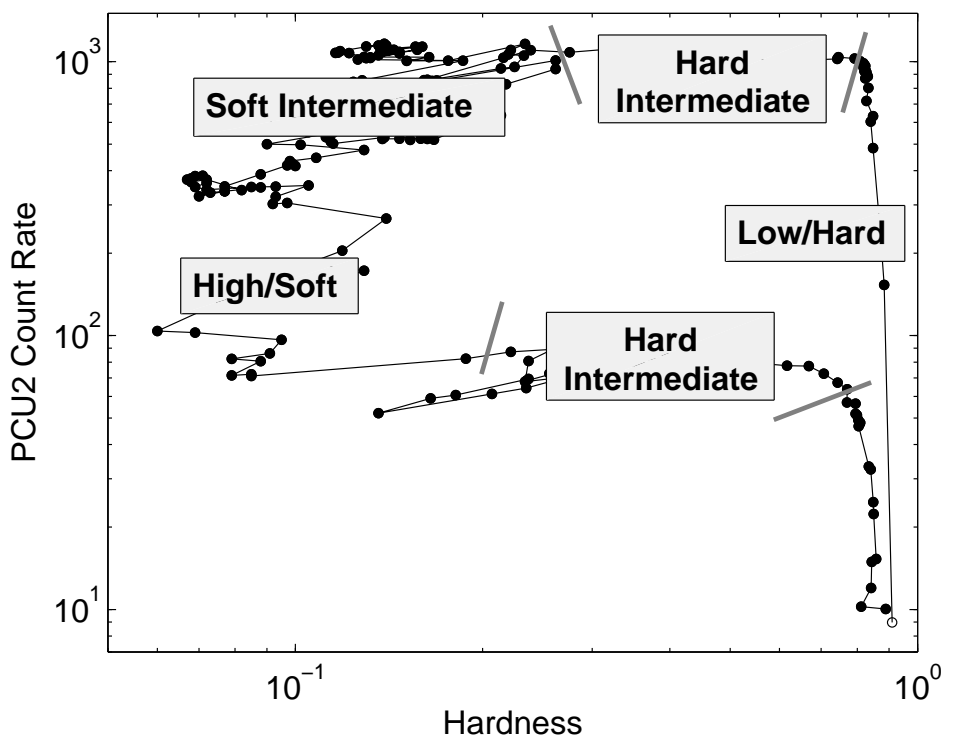

Figure 5. The HID of the 2002/2003 outburst of GX 339-4. Clear transitions are marked by gray segments. The branches corresponding to the four basic states in the q-track are labeled (Belloni et al., 2004).

properties that once were thought to be characteristic of the hard and hard intermediate states are still present in the soft state, although in a much weaker form.

\subsection{Discussion}

Although the picture presented here is somewhat simplified, as additional complications have been observed, we can sketch the states by using once more GX 339-4 as a template (see Fig. 5 and Fender et al., 2004). As shown above, the hard intermediate and soft intermediate states are kept separate, as both spectral and timing evolution show marked differences.

The hard state and the hard intermediate states have much in common. Spectrally, they are dominated by a hard component for which a high-energy cutoff is observed (Grove et al., 1998). In the timing domain, the components observed in the power density spectrum in these two states are clearly related, as can be seen from the evolution of their characteristic frequencies (Belloni et al., 2004). Nevertheless, something happens to the accretion flow as the source moves from the low/hard state to the intermediate state, as can be seen from multiwavelength studies (Homan et al., 2004a). This is likely to be related to 
changes in the jet, which could be responsible for part of the observed X-ray flux (Fender et al., 2004) as is also indicated by the fact that radio emission is always observed in these states.

The soft state and soft intermediate state are spectrally somewhat related. The energy spectrum is dominated by the thermal disk component, with a steep hard component with no evidence of a high-energy cutoff. The power density spectrum lacks band-limited components and shows only QPOs superimposed on a power-law component. No radio emission is observed (i.e. only upper limits on the emission from the compact source), indicating that the production of a jet is terminated at the transition to soft intermediate state (Fender et al., 2004) and maybe even before. During these states, the accretion flow is clearly different from the other two. In terms of variability however, the soft state shares some properties with the hard state and hard intermediate state, with the typical variability time scales following the same relation as seen in those states. If the hard state variability properties are linked to jet production, the presence of related (but much weaker) variability in the soft state could indicate a very weak (i.e. below current detection limits) jet in that state as well.

In addition to these general properties, there is a number of important topics whose detailed discussion is beyond the scope of the present paper. Most notably, the fast transitions observed between different states (never involving the hard state, which is only reserved for the beginning and the end of an outburst) need to be studied in detail, as they probably hold the key for a deeper understanding of the physics of the states and their association to the jet. Also, some sources can have bright outbursts without ever leaving the hard state, indicating that once again the instantaneous mass accretion rate is not what determines the transitions. However, the recent history of the mass accretion rate may play an important role (this can also be seen in Fig. 4, as GX 339-4 was observed to leave the hard state are very different flux levels). A secondary accretion flow (Smith et al., 2002; Yu et al., 2004) or changes in the Compton cooling/heating in the accretion disk corona (MeyerHofmeister et al., 2004) seem to be promising to explain some of these issues. For example, delays between a fast (i.e. in terms of propagation speed) secondary flow and a slower disk flow could account for the observed hysteresis.

In conclusion, the hysteresis behavior that can be seen from the HIDs in Figure 1, put forward for the first time by Miyamoto et al. (1995), was found with $R X T E$ to be a characteristic pattern for black-hole transients. Despite many complexities, general features have been shown to relate to the ejection of powerful relativistic jets, which can lead to a deeper understanding of the physical properties of the accretion flow. 
The picture presented here, scaled to longer time scales, should also be valid for AGNs. In these sources, there is no contribution of the optically thick accretion disk in the X-ray band, but the path shown in Fig. 5 is qualitatively similar if the disk contribution is removed. Recently, Cui (2004) presented a very similar diagram for the RXTE observations of the highly variable AGN Mkn 421, showing that indeed the scheme of accretion states could apply to systems over a large scales of masses.

JH wishes to thank Jon Miller and Ron Remillard for numerous discussions on the topic of black-hole states.

\section{References}

Bailyn, C. and L. Ferrara: 2004, 'GX 339-4: Continued Optical and Infra-red Decline'. The Astronomer's Telegram 323.

Belloni, T., J. Homan, P. Casella, M. van der Klis, E. Nespoli, W. Lewin, J. Miller, and M. Méndez: 2004, 'The evolution if the timing properties of the black-hole transient GX 339-4 durirng its 2002/2003 outburst'. Astronomy and Astrophysics p. submitted.

Belloni, T., M. Klein-Wolt, M. Méndez, M. van der Klis, and J. van Paradijs: 2000, 'A model-independent analysis of the variability of GRS 1915+105'. Astronomy and Astrophysics 355, 271-290.

Casella, P., T. Belloni, J. Homan, and L. Stella: 2004, 'A study of the low-frequency quasi-periodic oscillations in the X-ray light curves of the black hole candidate XTE J1859+226'. Astronomy and Astrophysics 426, 587-600.

Corbel, S., M. A. Nowak, R. P. Fender, A. K. Tzioumis, and S. Markoff: 2003, 'Radio/X-ray correlation in the low/hard state of GX 339-4'. Astronomy and Astrophysics 400, 1007-1012.

Cui, W.: 2004, 'X-ray Flaring Activity of Mrk 421'. astro-ph/0401222.

Esin, A. A., J. E. McClintock, and R. Narayan: 1997, 'Advection-dominated Accretion and the Spectral States of Black Hole X-Ray Binaries: Application to Nova MUSCAE 1991'. The Astrophysical Journal 489, 865.

Fender, R. P., T. M. Belloni, and E. Gallo: 2004, 'Towards a unified model for black hole X-ray binary jets'. Monthly Notices of the Royal Astronomical Society 355, 1105-1118.

Gallo, E., S. Corbel, R. P. Fender, T. J. Maccarone, and A. K. Tzioumis: 2004, 'A transient large-scale relativistic radio jet from GX 339-4'. MNRAS 347, L52-L56.

Grove, J. E., W. N. Johnson, R. A. Kroeger, K. McNaron-Brown, J. G. Skibo, and B. F. Phlips: 1998, 'Gamma-Ray Spectral States of Galactic Black Hole Candidates'. The Astrophysical Journal 500, 899.

Homan, J., M. Buxton, S. Markoff, C. Bailyn, E. Nespoli, and T. Belloni: 2004a, 'Multi-wavelength observations of the 2002 Outburst of GX 339-4: two patterns of X-ray-optical /near-infrared behavior'. The Astrophysical Journal p. submitted.

Homan, J., M. Klein-Wolt, S. Rossi, J. M. Miller, R. Wijnands, T. Belloni, M. van der Klis, and W. H. G. Lewin: 2003, 'High-Frequency Quasi-periodic Oscillations 
in the Black Hole X-Ray Transient XTE J1650-500'. The Astrophysical Journal 586, 1262-1267.

Homan, J., J. M. Miller, R. Wijnands, M. van der Klis, T. Belloni, D. Steeghs, and W. H. G. Lewin: 2004b, 'High- and low-frequency quasi-periodic oscillations in the X-ray light curves of the black hole transient H1743-322'. The Astrophysical Journal, in press, astro-ph/0406334.

Homan, J., R. Wijnands, M. van der Klis, T. Belloni, J. van Paradijs, M. KleinWolt, R. Fender, and M. Méndez: 2001, 'Correlated X-Ray Spectral and Timing Behavior of the Black Hole Candidate XTE J1550-564: A New Interpretation of Black Hole States'. The Astrophysical Journals 132, 377-402.

Kalemci, E., J. A. Tomsick, M. M. Buxton, R. E. Rothschild, K. Pottschmidt, S. Corbel, C. Brocksopp, and P. Kaaret: 2004, 'Multi-wavelength observations of the Galactic black hole transient 4U1543-47 during outburst decay: state transitions and jet contribution'. astro-ph/0409092.

Maccarone, T. J. and P. S. Coppi: 2003, 'Hysteresis in the light curves of soft X-ray transients'. MNRAS 338, 189-196.

McClintock, J. E. and R. A. Remillard: 2004, 'Black Hole Binaries'. astroph/0306213.

Meyer-Hofmeister, E., B. F. Liu, and F. Meyer: 2004, 'Hysteresis in spectral state transitions - a challenge for theoretical modeling'. astro-ph/0411145.

Miyamoto, S., S. Kitamoto, K. Hayashida, and W. Egoshi: 1995, 'Large hysteretic behavior of stellar black hole candidate X-ray binaries'. The Astrophysical Journall 442, L13-L16.

Remillard, R. A., G. J. Sobczak, M. P. Muno, and J. E. McClintock: 2002, 'Characterizing the Quasi-periodic Oscillation Behavior of the X-Ray Nova XTE J1550-564'. The Astrophysical Journal 564, 962-973.

Rossi, R., J. Homan, J. Miller, and T. Belloni: 2003, 'State transitions in the 2001/2002 outburst of XTE J1650-500'. astro-ph/0309129.

Smith, D. M., W. A. Heindl, and J. H. Swank: 2002, 'Two Different Long-Term Behaviors in Black Hole Candidates: Evidence for Two Accretion Flows?'. The Astrophysical Journal 569, 362-380.

Wijnands, R., J. Homan, and M. van der Klis: 1999, 'The Complex Phase-Lag Behavior of the 3-12 HZ Quasi-Periodic Oscillations during the Very High State of XTE J1550-564'. The Astrophysical Journall 526, 33.

Yu, W., M. van der Klis, and R. Fender: 2004, 'The Correlation between Hard X-Ray Peak Flux and Soft X-Ray Peak Flux in the Outburst Rise of Low-Mass X-Ray Binaries'. The Astrophysical Journal Letters 611, L121-L124. 
homan_belloni.tex; 15/08/2018; 19:54; p.14 